\title{
Sporadic childhood hepatoblastomas show activation of $\beta$-catenin, mismatch repair defects and p53 mutations
}

\author{
Maria C Curia ${ }^{1,2}$, Michele Zuckermann,4, Laura De Lellis ${ }^{1,2}$, Teresa Catalano ${ }^{5}$, \\ Rossano Lattanzio ${ }^{1,2}$, Gitana Aceto $^{1,2}$, Serena Veschi ${ }^{1,2}$, Alessandro Cama ${ }^{1,2}$, \\ Jean-Bernard Otte ${ }^{4}$, Mauro Piantelli ${ }^{1,2}$, Renato Mariani-Costantini ${ }^{1,2}$, Francesco Cetta ${ }^{3}$ \\ and Pasquale Battista ${ }^{2,6}$
}

${ }^{1}$ Departments of Oncology and Neurosciences, University of Chieti-Pescara, Chieti, Italy; ${ }^{2}$ Center of Excellence on Aging, 'Gabriele d'Annunzio' University Foundation, Chieti, Italy; ${ }^{3}$ Inter-University Center for Research in Hepatobiliary Disease, Department of Surgery, University of Siena, Siena, Italy; ${ }^{4}$ Liver Transplant Unit, Department of Surgery, University Saint-Luc of Louvain, Bruxelles, Belgium; ${ }^{5}$ Department of Experimental Pathology and Microbiology, University of Messina, Messina, Italy and ${ }^{6}$ Department of Human Movement Sciences, University of Chieti-Pescara, Chieti, Italy

\begin{abstract}
Hepatoblastoma, a rare embryonic tumor that may arise sporadically or in the context of hereditary syndromes (familial adenomatous polyposis and Beckwith-Wiedemann's) is the most frequent liver cancer of childhood. Deregulation of the APC/ $\beta$-catenin pathway occurs in a consistent fraction of hepatoblastomas, with mutations in the $A P C$ and $\beta$-catenin genes implicated in familial adenomatous polyposis-associated and sporadic hepatoblastomas, respectively. Alterations in other cancer-related molecular pathways have not been reported. We investigated a series of 21 sporadic paraffin-embedded hepatoblastoma cases for mutations in the p53 (exons 5-8) and $\beta$-catenin (exon 3 ) genes, loss of heterozygosity at $A P C$, microsatellite instability and immunohistochemical expression of $\beta$-catenin and of the two main mismatch repair proteins, MLH1 and MSH2. No loss of heterozygosity at $A P C$ was detected. We found mutations in $\beta$-catenin and $p 53$ in 4/21 (19\%) and $5 / 21$ $(24 \%)$ cases respectively, $\beta$-catenin protein accumulation in $14 / 21$ cases $(67 \%)$, microsatellite instability in $17 / 21$ cases $(\mathbf{8 1} \%)$, of which eight resulted positive for high-level of microsatellite instability (in four cases associated with loss of $\mathrm{MLH} 1 / \mathrm{MSH} 2$ immunostaining). No correlations between involved molecular pathway(s) and hepatoblastoma histotype(s) emerged. This study confirms that $\beta$-catenin deregulation is involved in sporadic hepatoblastoma and also suggests that mismatch repair defects and p53 mutations contribute to this rare liver cancer. Sporadic hepatoblastoma appears to be molecularly and phenotypically heterogeneous and may reflect different pathways of liver carcinogenesis.
\end{abstract}

Modern Pathology (2008) 21, 7-14; doi:10.1038/modpathol.3800977; published online 26 October 2007

Keywords: hepatoblastoma; mutations; $\beta$-catenin; $p 53$; microsatellite instability; immunohistochemistry

Hepatoblastoma, a rare embryonic tumor in the general population, represents the most frequent malignant liver tumor in childhood, with an incidence of one new case per 1 million under 15 years of age, male predominance,,$^{1}$ and median age at diagnosis of 1 year. Most hepatoblastomas develop sporadically, but the incidence of the disease rises up to $1000-2000$ folds in children from kindreds

Correspondence: Dr MC Curia, PhD, Department of Oncology and Neurosciences, Faculty of Medicine, University 'Gabriele d'Annunzio' of Chieti-Pescara, Via dei Vestini 31, Chieti 66013, Italy. E-mail: mc.curia@unich.it

Received 12 June 2007; revised 17 September 2007; accepted 19 September 2007; published online 26 October 2007 with familial adenomatous polyposis ${ }^{2}$ or BeckwithWiedemann syndrome. ${ }^{3}$

Several studies indicate that alterations in the APC/ $\beta$-catenin pathway, which result in Wnt signal activation, play a role in the pathogenesis of hepatoblastoma. In fact, double-hit inactivation of the $A P C$ gene has been demonstrated in familial adenomatous polyposis-associated hepatoblastoma. ${ }^{4-8}$ Furthermore, mutations affecting the phosphorylation sites encoded in exon 3 of $\beta$-catenin (a mutation hot-spot in several epithelial cancers) have been detected in sporadic hepatoblastoma, where $A P C$ mutations seem to be rare..$^{4-8}$

Somatic mutations in exons $5-8$ of the p53 gene, which encode the DNA-binding region where cancer-associated mutations most frequently 
occur, ${ }^{9-11}$ are strongly implicated in the progression of the common adult form of liver cancer, hepatocellular carcinoma. No hepatoblastoma-associated p53 mutations have been reported thus far, ${ }^{12}$ however the rare Li-Fraumeni syndrome, due to germline $p 53$ mutations, ${ }^{13,14}$ predisposes to a wide spectrum of early-onset cancers, and hepatoblastoma has been reported in Li-Fraumeni syndrome. ${ }^{13}$

Hepatoblastoma is not included in the spectrum of tumors associated with the hereditary nonpolyposis colorectal cancer syndrome, due to mismatch repair gene defects. However, reduced expression of the two main mismatch repair proteins, MLH1 and MSH2, and high-level microsatellite instability play a role in hepatocellular carcinoma. ${ }^{15-17}$ No data on microsatellite instability status and on expression of mismatch repair proteins are available for hepatoblastoma.

To shed further light on the molecular alterations associated with hepatoblastoma, we analyzed a series of 21 sporadic hepatoblastoma cases for loss of heterozygosity at the $A P C$ locus, mutations in the $\beta$-catenin and p53 genes, microsatellite instability and immunohistochemical expression of $\beta$-catenin, MLH1 and MSH2.

\section{Materials and methods}

\section{Patients and Tissue Samples}

We retrieved a retrospective series of 21 sporadic hepatoblastoma cases from pediatric patients who, between 1995 and 1999, underwent partial hepatectomy (eight cases) or liver transplantation (13 cases) at the Department of Surgery, Liver Transplant Unit, Louvain Catholic University, St Luc Hospital, Bruxelles, Belgium. The cases who underwent partial hepatectomy were given preoperative chemotherapy (cisplatin and doxorubicin) according to the protocols of the Sociéte Internationale d'Oncologie Pediatrique. ${ }^{18}$ Guardians of the patients gave written informed consent to biomedical studies after verbal counseling. Thirteen patients were male, 8 female; ages ranged from 2 to 132 months. None of the cases had family history of familial adenomatous polyposis, Li-Fraumeni syndrome, hereditary nonpolyposis colorectal cancer or other hereditary cancer syndromes, none was associated with hemihypertrophy or other congenital abnormalities suggestive of Beckwith-Wiedemann syndrome, ${ }^{19}$ and all resulted negative for hepatitis B virus infection. Cases were re-reviewed by a single pathologist (MP). Formalin-fixed/paraffin embedded tissue blocks were sectioned at $5 \mu \mathrm{m}$ and lightly stained with hematoxylin-eosin. Each block contained representative tumor areas together with non-neoplastic adjacent liver parenchyma. Tumor and non-tumor areas from 1 to 3 serial sections, identified using an hematoxylin-eosin stained section, were manually microdissected into $1.5 \mathrm{ml}$ polypropylene vials for DNA extraction, as described. ${ }^{20}$

\section{Loss of Heterozygosity and Microsatellite Instability Analyses}

For microsatellite typing we used a protocol consisting of a non-radioactive external PCR followed by a radioactive internal PCR, as described. ${ }^{20}$ Microsatellite instability was blindly scored by three investigators. Loss of heterozygosity at the APC locus was investigated in matched normal/tumor DNA analyzing seven microsatellites (D5S644, D5S492, D5S82, D5S299, D5S346, D5S656, D5S404) and the common APC intragenic polymorphism c.1458 (Y486Y), studied by RsaI restriction fragment length polymorphism analysis. ${ }^{21}$ Microsatellite instability was evaluated at the 5 loci recommended by the National Cancer Institute reference panel (BAT25, BAT26, D2S123, D3S1611, D5S346) ${ }^{22}$ Microsatellite instability at $\geq 2$ loci was defined as high-level (MSI-H), at $<2$ loci as low-level (MSI-L), cases in which no instability was detected were considered microsatellite-stable.

\section{Mutational Analyses}

$\beta$-Catenin exon 3 and p53 exons 5-8 were screened by single-strand conformational polymorphism analysis. ${ }^{20,23}$ Direct sequencing of the positive samples was performed using an ABI PRISM 310 Genetic Analyzer (Applied Biosystems, Foster City, CA, USA). Primers and PCR conditions are available upon requests. Mutations at $\beta$-catenin codon 32 were confirmed by HinfI restriction fragment-length polymorphism assay, where the 140 base pairs PCR product yields fragments of 73, 60 and 7 base pairs if wild type and of 80 and 60 base pairs if mutated. To exclude PCR artefacts, all mutations were confirmed on both DNA strands and in duplicate experiments on separately extracted DNA samples. Due to lack of frozen samples, we could not investigate large $\beta$-catenin gene deletions, which may occur in hepatoblastoma. ${ }^{5}$

\section{Immunohistochemistry}

For immunohistochemistry $5 \mu$ m-thick sections were stained with antibodies against MLH1 (BD Pharmingen, clone G168-15, diluted 1:25), MSH2 (Oncogene, clone FE11, diluted 1:100) and $\beta$-catenin (Novocastra, clone 17C2, diluted 1:100). Reactions were developed using the EnVision kit system (K4001, Dako, Glostrup, Denmark), with diaminobenzidine as chromogen. In control sections the specific primary antibody was omitted or replaced with non-immune serum or isotype-matched immunoglobulins (Dako). In one case, sections for immunohistochemistry were not available, due to exhaustion of the paraffin-embedded block. Cases were considered negative for MLH1 and/or MSH2 staining only in the presence of positive internal control (normal bile duct epithelium). 


\section{Results}

The institutional series of 21 sporadic hepatoblastoma cases collected between 1995 and 1999 at the Liver Transplant Unit of the Louvain Catholic University in Bruxelles was pathologically reviewed and subclassified according to Ishak et $a l^{24}$ as epithelial ( $n=11$, of which seven with fetal pattern and four with embryonal/fetal pattern) and mixed epithelial/mesenchymal $(n=10$, of which one is with teratoid features) (Table 1).

All cases were analyzed for loss of heterozygosity at the $A P C$ locus and were heterozygous at a minimum of four of eight tested markers. No evidence of loss of heterozygosity was found.

We detected three different $\beta$-catenin mutations in 4/21 cases (19\%), namely: a GAC $\rightarrow$ GGC transition at codon 32 (D32G) in cases \#4 and \#12; a GTT $\rightarrow$ ATT transition at codon 22 (V22I) in case \#19; a $\overline{\mathrm{CAA}} \rightarrow$ TAA nonsense mutation (Q27X) at codon 27 in case \#3. With regard to histotype, $\beta$-catenin mutations were detected in $2 / 11(18 \%)$ epithelial and $2 / 10(20 \%)$ mixed epithelial/mesenchymal cases (Tables 1 and 2). Immunohistochemistry revealed $\beta$-catenin immunostaining in $14 / 21$ cases $(67 \%)$, which included three of the four cases mutated in the $\beta$-catenin gene (Table 1, Figure 2a and b). Accumulation of $\beta$-catenin was detected in both epithelial $(8 / 11,73 \%)$ and mixed epithelial/ mesenchymal $(6 / 10,60 \%)$ cases, being preferentially localized in embryonal and mesenchymal areas, respectively (Table 1).

We detected 10 different p53 mutations in 5/21 cases (24\%; Tables 1 and 2). These included 1 missense mutation in exon 5 (T140I, case \#8); 1 nonsense mutation in exon 6 (Q192X, case \#8); 3 missense mutations in exon 7 (R248W, case \#3; H233Y, cases \#4 and \#8; C242Y, case \#13); 3 missense mutations in exon 8 (G279E, case \#3; E271K, case \#8; P292S, case \#13). In addition, we found two silent mutations in exon 7 (I251I, case \#4 and R249R, case \#16). Multiple p53 mutations were detected in cases \#8 (four mutations), \#3, \#4 and \#13 (two mutations each). With regard to histotype, p53 mutations were detected in $3 / 11$ (27\%) epithelial cases (of which two also mutated in $\beta$-catenin) and in $2 / 10(20 \%)$ mixed epithelial/mesenchymal cases (Table 1).

Microsatellite instability, assessed at the 5 loci recommended by the National Cancer Institute reference panel, ${ }^{22}$ was detected in $17 / 21$ cases (81\%) (Tables 1 and 2, Figure 1). According to Boland et al, ${ }^{22}$ eight cases were defined as high-level microsatellite instability, nine as low-level microsatellite instability, and four as microsatellite-stable. With regard to histotype, microsatellite instability was detected in 10/11 (91\%) epithelial cases, and in $7 / 10(70 \%)$ mixed epithelial/mesenchymal cases, of which five and three were high-level microsatellite instability, respectively.

We analyzed by immunohistochemistry MLH1 and MSH2 expression in seven of the eight high-level microsatellite instability-positive cases (one case could not be examined due to exhaustion of the block). Four cases showed loss of immunostaining: two resulted negative for MLH1, two revealed loss of both MLH1 and MSH2 (Tables 1 and 2, Figure 2c-f).

A possible role of preoperative chemotherapy in determining the detected hepatoblastoma-associated molecular alterations is unlikely, as mutations in $\beta$-catenin, $p 53$, and microsatellite instability as well as alterations in $\beta$-catenin and MLH1 and MSH2 immunostaining were observed in both treated and untreated cases (Tables 1 and 2).

\section{Discussion}

Little is known about the molecular pathways that contribute to hepatoblastoma. ${ }^{4}$ We studied a series of 21 sporadic hepatoblastomas collected at the Louvain Catholic University in Bruxelles. The cases were analyzed for loss of heterozygosity at the $A P C$ locus, mutations in the $\beta$-catenin, and p53 genes, $\beta$-catenin protein accumulation and microsatellite instability status. Cases determined as high-level microsatellite instability were further characterized for the expression of the MLH1 and MSH2 proteins by immunohistochemistry.

Absence of loss of heterozygosity at the $A P C$ locus confirms literature reports suggesting that $A P C$ gene alterations are not implicated in sporadic hepatoblastoma. ${ }^{7,25}$

Mutational analysis of $\beta$-catenin exon 3 , which encodes a region rich in serine and threonine residues that serve as targets of phosphorylation by GSK-3 $\beta,^{8}$ revealed point mutations in $4 / 21$ (19\%) cases. These mutations may influence $\beta$-catenin stability. ${ }^{5,26}$

The detection of $\beta$-catenin mutations and/or protein accumulation in the majority of our hepatoblastoma cases $(15 / 21 ; 71 \%)$ confirms that Wnt signal activation is implicated in the pathogenesis of sporadic hepatoblastoma. ${ }^{5-7}$ Our results are in agreement with the studies of Koch et $a l^{5,27}$ who detected $\beta$-catenin point mutations in 22 and $25 \%$ of their sporadic hepatoblastoma cases, while Taniguchi et al reported a slightly higher percentage (33\%) of mutated cases. ${ }^{28}$ As reported by Takayasu et al, ${ }^{25}$ we observed that the hepatoblastoma cases with $\beta$-catenin protein accumulation were much more numerous than the cases with identified $\beta$-catenin mutations. In this respect it should be noted that we could not investigate large $\beta$-catenin gene deletions, which are known to occur in hepatoblastoma. ${ }^{5,27,28}$ Furthermore, we cannot exclude alterations in Wnt regulators acting upstream of $\beta$-catenin ${ }^{29}$ such as AXIN1 and AXIN2, implicated in hepatoblastoma cases negative for mutations in the $\beta$-catenin gene but positive for $\beta$-catenin protein accumulation. ${ }^{28,30}$ With regard to correlations with histology, it is noteworthy that $\beta$-catenin immunostaining tended 
Table 1 Clinical-pathological characteristics, $\beta$-catenin and p53 gene mutations, microsatellite instability status and expression of $\beta$-catenin, MLH1 and MSH2 in 21 sporadic hepatoblastomas

\begin{tabular}{|c|c|c|c|c|c|c|c|c|c|c|c|c|c|c|c|c|}
\hline \multirow[t]{2}{*}{ Histotype (n) } & \multirow[t]{2}{*}{ Case \# } & \multirow[t]{2}{*}{$\begin{array}{c}\text { Age }{ }^{a} \\
\text { (months) }\end{array}$} & \multirow[t]{2}{*}{ Sex } & \multirow[t]{2}{*}{$\begin{array}{l}\beta \text {-catenin } \\
\text { mutation }\end{array}$} & \multicolumn{3}{|c|}{$\beta$-catenin expression ${ }^{\mathrm{b}}$} & \multirow[t]{2}{*}{ p53 mutation } & \multicolumn{5}{|c|}{ MSI status ${ }^{\mathrm{C}}$} & \multirow[t]{2}{*}{ MSI } & \multicolumn{2}{|c|}{$\begin{array}{c}\text { MMR } \\
\text { expression }^{\mathrm{d}}\end{array}$} \\
\hline & & & & & $\begin{array}{c}\text { Fetal } \\
\text { area }\end{array}$ & $\begin{array}{l}\text { Embryonal } \\
\text { area }\end{array}$ & $\begin{array}{c}\text { Mesenchymal } \\
\text { area }\end{array}$ & & D2S123 & D3S1611 & $D 5 S 346$ & BAT25 & BAT26 & & MLH1 & MSH2 \\
\hline \multicolumn{17}{|l|}{ Epithelial } \\
\hline \multirow[t]{7}{*}{ (a) Fetal pattern (7) } & 1 & $60^{\mathrm{a}}$ & M & No & No & & & No & + & - & + & - & - & MSI-H & NA & NA \\
\hline & 2 & $67^{\mathrm{a}}$ & M & No & M C & & & No & - & + & - & - & - & MSI-L & & \\
\hline & 3 & $130^{\mathrm{a}}$ & M & Q27X & M C & & & $\begin{array}{l}\text { R248W, } \\
\text { G279E }\end{array}$ & + & - & - & - & - & MSI-L & & \\
\hline & 4 & $44^{\mathrm{a}}$ & $\mathrm{M}$ & D32G & $\mathrm{N}$ & & & H233Y, I251I & + & + & - & - & - & MSI-H & No & No \\
\hline & 5 & 4 & $\mathrm{~F}$ & No & $\mathrm{M}$ & & & No & + & - & - & - & - & MSI-L & & \\
\hline & 6 & 5 & $\mathrm{~F}$ & No & No & & & No & + & + & + & - & + & MSI-H & No & $\mathrm{N}$ \\
\hline & 7 & 3 & $\mathrm{~F}$ & No & No & & & No & + & - & - & - & - & MSI-L & & \\
\hline \multirow[t]{4}{*}{$\begin{array}{l}\text { (b) Embryonal/ } \\
\text { fetal pattern (4) }\end{array}$} & 8 & 2 & $\mathrm{~F}$ & No & No & $\mathrm{N}$ & & $\begin{array}{l}\text { Q192X,T140I, } \\
\text { H233Y, } \\
\text { E271K }\end{array}$ & + & - & + & - & - & MSI-H & $\mathrm{N}$ & $\mathrm{N}$ \\
\hline & 9 & $39^{\mathrm{a}}$ & $\mathrm{F}$ & No & No & $\mathrm{C} \mathrm{N}$ & & No & - & - & - & - & - & MSS & & \\
\hline & 10 & 9 & $\mathrm{~F}$ & No & No & $\mathrm{N}$ & & No & + & - & + & - & - & MSI-H & $\mathrm{N}$ & $\mathrm{N}$ \\
\hline & 11 & $29^{\mathrm{a}}$ & M & No & No & $\mathrm{CN}$ & & No & + & - & - & - & - & MSI-L & & \\
\hline \multicolumn{17}{|c|}{ Epithelial/mesenchymal } \\
\hline \multirow{9}{*}{ (a) Not teratoid (9) } & 12 & 15 & M & D32G & No & No & No & No & - & - & - & - & + & MSI-L & & \\
\hline & 13 & 3 & M & No & $\mathrm{N}$ & No & M C & C242Y, P292S & - & - & - & - & - & MSS & & \\
\hline & 14 & 5 & $\mathrm{~F}$ & No & No & No & M & No & - & - & - & - & - & MSS & & \\
\hline & 15 & 3 & M & No & No & $\mathrm{CN}$ & M C N & No & + & - & - & + & - & MSI-H & No & $\mathrm{N}$ \\
\hline & 16 & 14 & M & No & M C & No & No & R249R & + & - & - & - & - & MSI-L & & \\
\hline & 17 & 22 & M & No & No & No & $\mathrm{M}$ & No & + & + & - & - & - & MSI-H & No & No \\
\hline & 18 & 13 & $\mathrm{~F}$ & No & No & No & No & No & - & - & & - & - & MSS & & \\
\hline & 19 & 8 & M & V22I & No & No & $\mathrm{N}$ & No & + & - & + & - & + & MSI-H & $\mathrm{N}$ & $\mathrm{N}$ \\
\hline & 20 & $45^{\mathrm{a}}$ & M & No & No & No & No & No & + & - & - & - & - & MSI-L & & \\
\hline (b) Teratoid (1) & 21 & $132^{\mathrm{a}}$ & M & No & No & No & No & No & + & - & - & - & - & MSI-L & & \\
\hline
\end{tabular}

Abbreviations: F, female; M, male; MMR, mismatch repair; MSI, microsatellite instability; NA, not available; No, no mutation or no protein expression.

${ }^{\mathrm{a}}$ Patients treated with preoperative chemotherapy.

${ }^{\mathrm{b}}$ Membranous (M), cytoplasmic (C), or nuclear (N) immunostaining.

${ }^{\mathrm{c}}$ Presence (+) or absence (-) of microsatellite instability.

${ }^{\mathrm{d}}$ Nuclear (N) immunostaining. 
Table 2 Status of $\beta$-catenin, p53 and microsatellite instability according to histotype in 21 sporadic hepatoblastomas

\begin{tabular}{|c|c|c|c|c|c|}
\hline & \multirow[t]{2}{*}{ All cases $\mathrm{n}=21$} & \multicolumn{2}{|c|}{ Pre-operative chemotherapy } & \multirow[t]{2}{*}{ Epithelial $\mathrm{n}=11$} & \multirow[t]{2}{*}{ Epithelial/mesenchymal $\mathrm{n}=10$} \\
\hline & & Yes, $\mathrm{n}=8$ & No, $\mathrm{n}=13$ & & \\
\hline \multicolumn{6}{|l|}{$\beta$-Catenin } \\
\hline Mutation (\%) & $4(19)$ & $2(25)$ & $2(15)$ & $2(18)$ & $2(20)$ \\
\hline Accumulation (\%) & $14(67)$ & $5(63)$ & $9(69)$ & $8(73)$ & $6(60)$ \\
\hline p53 Mutations (\%) & $5(24)$ & $2(25)$ & $3(23)$ & $3(27)$ & $2(20)$ \\
\hline $\operatorname{MSI}(\%)$ & $17(81)$ & & & $10(91)$ & $7(70)$ \\
\hline MSI-H (\%) & $8(38)$ & $2(25)$ & $6(46)$ & $5(45)$ & $3(30)$ \\
\hline MSI-L (\%) & $9(43)$ & $5(62)$ & $4(31)$ & $5(45)$ & $4(40)$ \\
\hline
\end{tabular}

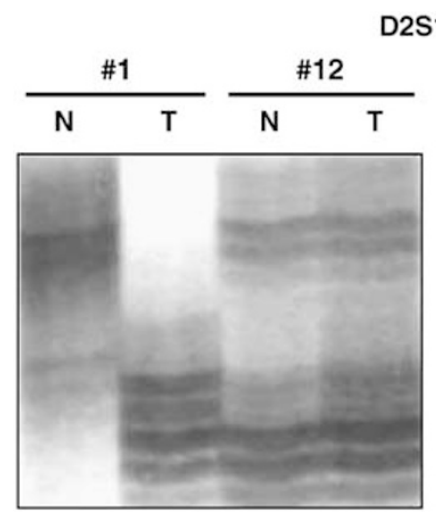

$\mathrm{DS} 123$
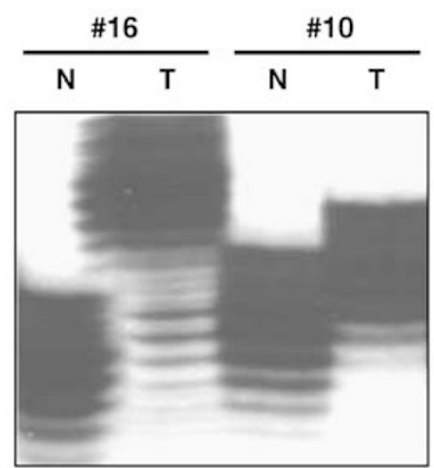
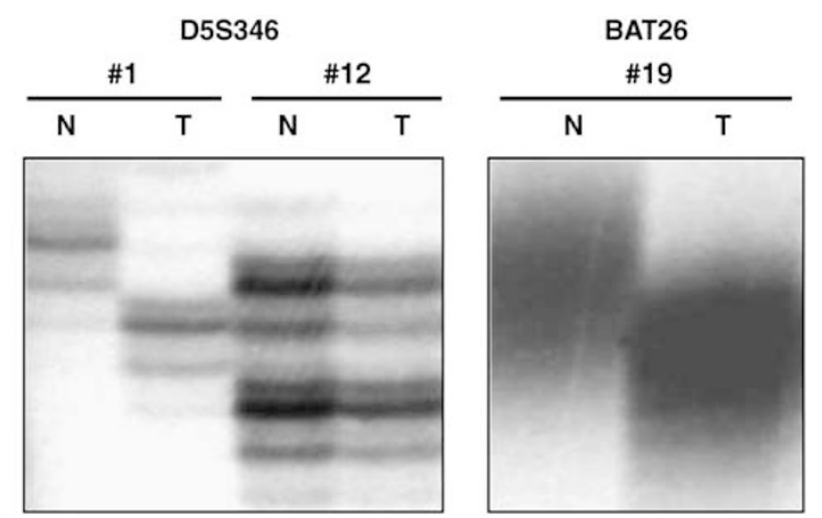

Figure 1 Examples of microsatellite instability typings at D2S123, D5S346 and BAT26 in five hepatoblastoma cases (N: normal; T: tumor): case \#1 shows microsatellite instability at D2S123 and D5S346; cases \#16 and \#10 show microsatellite instability at D2S123; case \#12 does not show microsatellite instability at the tested loci; case \#19 shows microsatellite instability at BAT26.

to be more evident in embryonal and in mesenchymal areas in epithelial and in mixed epithelial/ mesenchymal hepatoblastoma, respectively. This is concordant with evidence suggesting that $\beta$-catenin activation interferes with developmental signals regulating early stages of hepatic differentiation. ${ }^{7,25}$

Literature data indicate that $p 53$ mutations occur commonly in the progression of tumors with Wntsignaling activation. ${ }^{31,32}$ The report of hepatoblastoma in two Li-Fraumeni syndrome patients is thus far the only evidence suggesting an involvement of p53 in hepatoblastoma. ${ }^{13}$ In the present study, we found somatic p53 mutations in 5/21 hepatoblastoma cases $(24 \%)$ and four of these cases harbored multiple p53 mutations. All detected mutations are listed in the International Agency for Cancer Research p53 mutation database (http:// www.iarc.fr/p53) in association with a variety of tumors. Interestingly, p53 Q192X and p53 C242Y, detected in two different hepatoblastoma cases, were previously found in hepatocellular carcinoma. Furthermore, p53 I251I, a silent mutation found in an hepatoblastoma case, was reported in both hepatitis B virus-positive and -negative hepatocellular carcinoma and also in hepatocellular carcinoma associated to aflatoxin exposure. The presence of somatic p53 mutations in hepatoblastoma suggests a possible role of pre- or peri-natal exposure to mutagens. Unfortunately, no information on course of pregnancy and weight at birth could be retrieved for our hepatoblastoma case series. It is also intriguing that we found microsatellite instability in the vast majority of our sporadic hepatoblastoma cases. In fact exposure to environmental toxicants/ carcinogens, particularly heavy metals, is correlated with microsatellite instability in adult cancers due to occupational exposure. ${ }^{33,34}$ In our hepatoblastoma series the high- and low-level microsatellite instability phenotypes did not appear to be specifically associated with $\beta$-catenin alterations, $p 53$ mutations, and epithelial vs epithelial/mesenchymal histology. A role of preoperative chemotherapy in determining the observed genetic alterations is unlikely, as mutations and microsatellite instability were found in both preoperatively-treated and -untreated hepatoblastoma cases.

The role of microsatellite instability in liver cancer is still debated. Microsatellite instability has been reported in hepatocellular carcinoma arising in the context of chronic hepatitis and cirrhosis and also in non-fibrotic hepatocellular carcinoma. ${ }^{16,17}$ Previous studies indicate that 

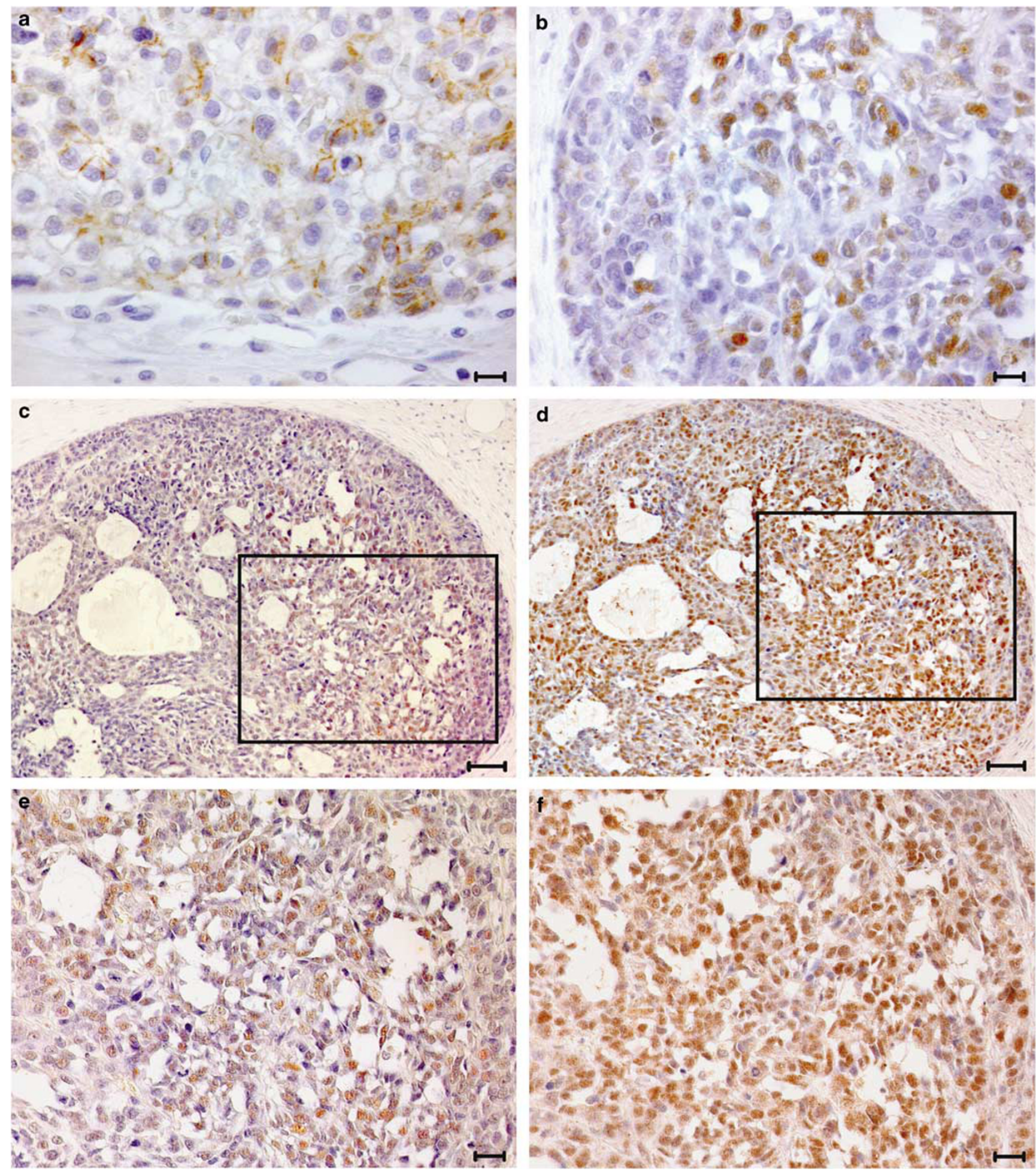

Figure 2 (a) Epithelial type hepatoblastoma with fetal pattern and $\beta$-catenin cell membrane staining (scale bar, $20 \mu \mathrm{m}$ ). (b) Epithelial hepatoblastoma with embryonal/fetal pattern and $\beta$-catenin nuclear staining in the embryonal area (scale bar, $20 \mu \mathrm{m})$. (c and d) Epithelialtype hepatoblastoma with embryonal/fetal pattern showing positive MLH1 and MSH2 immunostaining (scale bars, $50 \mu \mathrm{m}$ ). (e and f) Higher-magnification views of insets in c and d, detailing nuclear expression of MLH1 (e) and MSH2 (f) in the embryonal area (scale bars, $20 \mu \mathrm{m})$.

reduced expression of the two major mismatch repair proteins, MLH1 and MSH2, occurs in hepatocellular carcinoma, particularly in association with hepatitis $\mathrm{C}$ virus infection. ${ }^{15}$ We found micro- satellite instability in the vast majority of our sporadic hepatoblastoma cases. The high-level and low-level microsatellite instability phenotypes did not appear to be specifically associated with 
$\beta$-catenin alterations, p53 mutations and epithelial vs epithelial/mesenchymal histology. While lowlevel microsatellite instability could reflect inherent repeat instability and clonal expansion, high-level microsatellite instability, particularly when associated with loss of MLH1/MSH2 staining (as in four of our hepatoblastoma cases) suggests that mismatch repair defects could play a pathogenetic role.

In conclusion our study suggests that $\beta$-catenin activation, $p 53$ gene mutations and loss of mismatch repair function may contribute, either independently or in synergy, to the development of sporadic hepatoblastoma. The molecular alterations affecting these pathways do not appear to correlate with clinical-pathological features, with the exception of preferential $\beta$-catenin accumulation in embryonal and mesenchymal areas. Sporadic hepatoblastoma seems to represent a molecularly heterogeneous cancer, which could reflect different molecular mechanisms of progression.

\section{Acknowledgement}

Study supported by funds from the Italian Ministry of University and Research and by a Biotransplant Fellowship, Boston, MA, USA.

\section{Conflict of interest}

None to declare.

\section{References}

1 Weinberg AG, Finegold MJ. Primary hepatic tumours of childhood. Hum Pathol 1983;14:512-537.

2 Hughes LJ, Michels VV. Risk of hepatoblastoma in familial adenomatous polyposis. Am J Med Genet 1992;43:1023-1025.

3 DeBaun MR, Tucker MA. Risk of cancer during the first four years of life in children from The BeckwithWiedemann Syndrome Registry. J Pediatr 1998;132: 398-400.

4 Buendia MA. Genetic alterations in hepatoblastoma and hepatocellular carcinoma: common and distinctive aspects. Med Pediatr Oncol 2002;39:530-535.

5 Koch A, Denkhaus D, Albrecht S, et al. Childhood hepatoblastomas frequently carry a mutated degradation-targeting box of the $\beta$-catenin gene. Cancer Res 1999;59:269-273.

6 Blaker H, Hofmann WJ, Rieker RJ, et al. Beta-catenin accumulation and mutation of the CTNNB1 gene in hepatoblastoma. Genes Chromosomes Cancer 1999;25: 399-402.

7 Wei Y, Fabre M, Branchereau S, et al. Activation of beta-catenin in epithelial and mesenchymal hepatoblastomas. Oncogene 2000;19:498-504.

8 Polakis P. Wnt signaling and cancer. Genes dev 2000;14:1837-1851.

9 Vogelstein B, Fearon ER, Hamilton SR, et al. Genetic alterations during colorectal-tumor development. N Engl J Med 1988;319:525-532.
10 Cho KR, Vogelstein B. Genetic alterations in the adenoma-carcinoma sequence. Cancer 1992;70(Suppl): 1727-1731.

11 Goh HS, Yao J, Smith DR. p53 point mutation and survival in colorectal cancer patients. Cancer Res 1995; 55:5217-5221.

12 Debuire B, Paterlini P, Pontisso P, et al. Analysis of the p53 gene in European hepatocellular carcinomas and hepatoblastomas. Oncogene 1993;8:2303-2306.

13 Nichols KE, Malkin D, Garber JE, et al. Germ-line p53 mutations predispose to a wide spectrum of early-onset cancers. Cancer Epidemiol 2001; 10:83-87.

14 Birch JM, Alston RD, McNally RJQ, et al. Relative frequency and morphology of cancers in carriers of germline TP53 mutations. Oncogene 2001;20: 4621-4628.

15 Zekri A-RN, Sabry GM, Bahnassy AA, et al. Mismatch repair genes (hMLH1, hPMS1, hPMS2, GTBP/hMSH6, hMSH2) in the pathogenesis of hepatocellular carcinoma. World J Gastroenterol 2005;11:3020-3026.

16 Salvucci M, Lemoine A, Saffroy R, et al. Microsatellite instability in European hepatocellular carcinoma. Oncogene 1999;18:181-187.

17 Chiappini F, Gross-Goupil M, Saffroy R, et al. Microsatellite instability mutator phenotype in hepatocellular carcinoma in non-alcoholic and non-virally infected normal livers. Carcinogenesis 2004;25: 541-547.

18 Pritchard J, Brown J, Shafford E, et al. Cisplatin, doxorubicin, and delayed surgery for childhood hepatoblastoma: a successful approach-results of the first prospective study of the International Society of Pediatric Oncology. J Clin Oncol 2000;18: 3819-3828.

19 Hamada Y, Takada K, Fukunaga S, et al. Hepatoblastoma associated with Beckwith-Wiedemann syndrome and hemihypertrophy. Pediatr Surg Int 2003;19: 112-114.

20 Curia MC, Palmirotta R, Aceto G, et al. Unbalanced germ-line expression of hMLH1 and hMSH2 alleles in hereditary nonpolyposis colorectal cancer. Cancer Res 1999;59:3570-3575.

21 Kraus C, Ballhausen G. Two intragenic polymorphisms of the APC gene detected by PCR and enzymatic digestion. Hum Genet 1992;88:705-706.

22 Boland CR, Thibodeau SN, Hamilton SR, et al. A National Cancer Institute Workshop on microsatellite instability for cancer detection and familial predisposition: development of international criteria for the determination of microsatellite instability in colorectal cancer. Cancer Res 1998;58:5248-5257.

23 Catalano T, Curia MC, Aceto G, et al. Mutations in the p53 and Ki-ras genes, microsatellite instability and site of tumor origin in colorectal cancer. Oncol Rep 2005;14:625-631.

24 Ishak KG, Goodman ZD, Stocker JT. Tumors of the liver and intrahepatic bile ducts. In: AFIP Atlas of Tumor Pathology, Third Series, Fascicle 31 (chapter 6, pg. 159-183). Armed Forces Institute of Pathology: Washington, DC, 2001.

25 Takayasu H, Horie H, Hiyama E, et al. Frequent deletions and mutations of the beta-catenin gene are associated with overexpression of cyclin D1 and fibronectin and poorly differentiated histology in childhood hepatoblastoma. Clin Cancer Res 2001;7: 901-908. 
26 Aberle H, Bauer A, Stappert J, et al. Beta-catenin is a target for the ubiquitin-proteasome pathway. EMBO J 1997;16:3797-3804.

27 Koch A, Waha A, Hartmann W, et al. Elevated expression of Wnt antagonists is a common event in hepatoblastomas. Clin Cancer Res 2005;11:4295-4304.

28 Taniguchi K, Roberts LR, Aderca IN, et al. Mutational spectrum of $\beta$-catenin, AXIN1, and AXIN2 in hepatocellular carcinomas and hepatoblastomas. Oncogene 2002;21:4863-4871.

29 Katoh M, Katoh M. WNT signaling pathway and stem cell signaling network. Clin Cancer Res 2007;13:4042-4045.

30 Koch A, Weber N, Waha A, et al. Mutations and elevated transcriptional activity of conductin (AXIN2) in hepatoblastomas. J Pathol 2004;204:546-554.
31 Damalas A, Kahan S, Shtutman M, et al. Deregulated $\beta$-catenin induces a p53- and ARF-dependent growth arrest and cooperates with Ras in transformation. EMBO J 2001;20:4912-4922.

32 Harris SL, Levine AJ. The p53 pathway: positive and negative feedback loops. Oncogene 2005;24: 2899-2908.

33 Hartwig A. Carcinogenicity of metal compounds: possible role of DNA repair inhibition. Toxicol Lett 1998;102:235-239.

34 Takahashi Y, Kondo K, Hirose T, et al. Microsatellite instability and protein expression of the DNA mismatch repair gene, hMLH1, of lung cancer in chromate-exposed workers. Mol Carcinog 2005;42: 150-158. 